\title{
Time of Therapeutic Procedure
}

National Cancer Institute

\section{Source}

National Cancer Institute. Time of Therapeutic Procedure. NCI Thesaurus. Code C162168.

The time that a specific therapeutic procedure was performed. 